\title{
On the Accuracy of DFT for Describing Hydrogen Bonds: Dependence on the Bond Directionality
}

\author{
Joel Ireta, ${ }^{*, \dagger}$ Jörg Neugebauer, ${ }^{\dagger, *}$ and Matthias Scheffler ${ }^{\dagger}$ \\ Fritz-Haber-Institut der Max-Planck-Gesellschaft, Faradayweg 4-6, D-14195 Berlin-Dahlem, Germany, and \\ Theoretische Physik, Universität Paderborn, D-33098 Paderborn, Germany
}

Received: December 4, 2003; In Final Form: April 6, 2004

\begin{abstract}
A set of representative hydrogen bonded dimers has been studied employing density functional theory (DFT) in the Perdew, Burke, and Ernzerhof (PBE) generalized gradient approximation. Our results for hydrogen bond (hb) strengths and geometry parameters show good agreement with those obtained by Møller-Plesset (MP2) or Coupled-Cluster (CC) methods. We observe that the reliability of DFT-PBE for the description of hbs is closely connected to the bond directionality (i.e. the angle between $\mathrm{D}-\mathrm{H}$ and $\mathrm{H} \cdots \mathrm{A}$ where $\mathrm{D}$ and A are the donor and the acceptor atoms or regions, respectively, in the hb interaction): with increasing deviation from a linear $\mathrm{D}-\mathrm{H} \cdots \mathrm{A}$ arrangement the accuracy of the DFT-PBE decreases.
\end{abstract}

\section{Introduction}

The hydrogen bond $(\mathrm{hb})$ interaction, $\mathrm{D}-\mathrm{H} \cdots \mathrm{A}$, is realized by the attractive force between a polar $\mathrm{D}-\mathrm{H}$ bond and a polar atom or region A (acceptor). Common (hydrogen) donor (D) and acceptor (A) atoms in such bonds are $\mathrm{C}, \mathrm{N}, \mathrm{O}$, and $\mathrm{F}$. According to their interaction energy hbs are often classified as strong ( $>15 \mathrm{kcal} / \mathrm{mol})$, moderate ( 4 to $15 \mathrm{kcal} / \mathrm{mol})$, and weak $(<4 \mathrm{kcal} / \mathrm{mol}) .{ }^{1}$ Hydrogen bonds play a crucial role in a wide range of materials, e.g., biomolecules, liquids, and molecular crystals. Consequently a large number of experimental and theoretical studies have been devoted to investigate this fundamental interaction. Nevertheless, its nature and strength is still not fully understood and in fact is still controversial. ${ }^{2,3}$ An important characteristic of hbs is its directionality, i.e., the angle $\theta$ between the $\mathrm{D}-\mathrm{H}$ and $\mathrm{H} \cdots \mathrm{A}$ bonds (see Figure 1). Usually $\theta$ is in the range from $140^{\circ}$ to $180^{\circ},{ }^{1}$ where typically smaller $\theta$ values go together with a weaker hb strength. For smaller angles the hb is believed to be largely due to the van der Waals (vdW) interaction., ${ }^{4,5}$

Determining the strength and geometry of hbs is a challenge, for experimental as well for theoretical studies. ${ }^{3,6}$ Regarding theoretical methods it is well-established that ab initio techniques accounting for electron correlation are needed for an accurate description of hbs, i.e., to ensure error bars of the hb strength of $1 \mathrm{kcal} / \mathrm{mol}$ or less. ${ }^{7,8}$ Hartree-Fock calculations are wellknown to underestimate the strength of hbs. This is corrected by using methods which explicitly include correlation such as second-order Møller-Plesset (MP2), coupled clusters (CC), or configuration interaction (CI) methods. Another crucial item for achieving an accurate description of hbs is to employ large (high quality) basis sets to expand the wave functions. ${ }^{9}$ This, together with the fact that correlated methods have to be used make an accurate description of hb systems computationally very demanding. To overcome this problem it is crucial to develop strategies that are computationally less demanding but nevertheless describe hbs with a similar accuracy as MP2 (or higher levels of theory). Obviously as a first step one has to check the

\footnotetext{
$\dagger$ Fritz-Haber-Institut der Max-Planck-Gesellschaft.

$\doteqdot$ Theoretische Physik, Universität Padeborn.
}

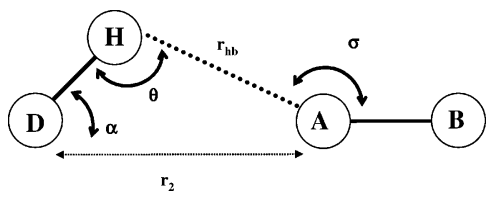

Figure 1. Parameters for describing the hb geometry.

range of validity of such alternative strategies, and this is the topic of this paper.

Density functional theory (DFT) is an obvious candidate. The accuracy of DFT to describe the hb interaction relies highly on the specific functional used to approximate the electronic exchange and correlation (xc) contributions. Some general trends concerning the performance/quality of the various xc functionals are known: (i) The local-density approximation (LDA) overestimates the hb strength. ${ }^{10,11}$ (ii) The generalized gradient approximations (GGA) and hybrid functionals are more accurate to describe the hbs than LDA. ${ }^{9-17}$ (iii) The most commonly used GGAs to treat hbs, the Becke exchange functional and the Lee-Yang-Parr functional for correlation (BLYP) ${ }^{18}$ and the Becke hybrid functional for exchange and the Lee-YangParr functional for correlation (B3LYP) ${ }^{19}$ commonly underestimate the hb strength with respect to MP2 or CC results. ${ }^{914,17,20,21}$ These trends apply for all systems studied in this work and also for others with similar hbs (see e.g. Rappé and Bernstein ${ }^{9}$ and Tsuzuki and Lüthi $^{20}$ ). More recently also other GGAs such as Perdew-Wang (PW91), ${ }^{22}$ the Becke approximation for exchange ${ }^{23}$ and the Perdew expression for correlation ${ }^{24}$ (BP), or the Perdew, Burke, and Ernzerhof (PBE) ${ }^{25}$ functional have been applied to study hbs. ${ }^{11,13,15,16,20,26-30}$ (iv) The reliability of more recently developed xc functionals that include the dependence on the laplacian of the density ${ }^{12,31}$ and/or the kinetic energy density ${ }^{32}$ is not known at all. (v) GGAs systematically provide too low classical energy barriers for proton transfer between donor and acceptor atoms. ${ }^{33}$

Besides errors in the hb description due to the $\mathrm{xc}$ functional, one also must consider errors associated with the basis-set expansion of the Kohn-Sham (KS) orbitals. There are three kinds of basis sets currently used: (i) localized Gaussian orbitals, (ii) localized numerical orbitals (here numerical stands only for 
the radial part of the orbital), and (iii) plane waves. Basis sets that may be called hybrids of numerical orbitals and plane waves, as, e.g., linear muffin tin orbitals, augmented plane waves, have not been used so far as they are numerically even more demanding. Gaussian orbitals are by much the most widely used in hb studies. However, these basis sets have a rather large basis set superposition error (BSSE) that must be (and typically is) corrected to obtain reliable hb interaction energies. ${ }^{34}$ The BSSE is a spurious contribution to the interaction energy arising from the improved description of the fragment $A$ in the complex $\mathrm{A} \cdots \mathrm{B}$ due to the assistance of the basis set located in $\mathrm{B}$, and vice versa. The counterpoise correction method is usually applied to correct BSSE. ${ }^{35}$ This technique corrects the interaction energy of the complex $\mathrm{A} \cdots \mathrm{B}$ by computing the energies of the isolated fragments A and B with exactly the same basis set (both in number and location) as used to compute the complex A $\cdots$ B. However, the accuracy of the counterpoise method to correct BSSE has been subject to some debate. ${ }^{34}$ Extrapolation of the interaction energy to the complete basis set limit is an alternative way for getting accurate interaction energies. It has been shown that counterpoise corrected and uncorrected interaction energies converge to the same value at the basis set limit (see e.g. Feyereisen et al. ${ }^{36}$ ).

Numerical orbitals appear to have very small BSSE. ${ }^{37,38}$ Yet they have not been extensively used to treat hydrogen-bonded systems. Plane-wave basis sets are mainly employed to study condensed matter systems. They are used together with the pseudopotential approach and periodic boundary conditions to solve the KS equations. ${ }^{39,40}$ Plane waves are inherently free of the $\mathrm{BSSE}^{41}$ since they are completely unbiased with respect to the atomic structure and in contrast to atomic orbitals they are not centered on the atomic nuclei thus describing any point in the periodic supercell with the same quality. Therefore, in contrast to atom-centered orbitals, fragments $\mathrm{A}$ or $\mathrm{B}$ are described with the same basis set independently whether they are isolated or in the complex A $\cdots \mathrm{B}$ (assuming of course the calculations are carried out with a unit cell of the same size and with the same plane-wave basis set energy cutoff-see computational aspects below).

In this work we study a set of hydrogen-bonded dimers using DFT-PBE. The purpose of this work is to establish a firm quantitative measure of the performance of the DFT-PBE functional to describe hbs in diverse geometric environments. We use a plane-wave basis set to describe the KS orbitals.

As a first step we have studied a set of small hydrogen-bonded systems (see Figure 2), which consist of dimers containing up to eight atoms to benchmark the accuracy of DFT-PBE against that of CC studies reported in the literature. In a second step we will focus on formamide (fm, Figure 3), $N$-methylacetamide (nma, Figure 4), and $N, N$-dimethylformamide (dmf, Figure 5) hydrogen-bonded dimers. These molecules form diverse hydrogenbonded conformers where the hb directionality varies between linear and highly bent. Thus, these molecules are well-suited to study the accuracy of our DFT calculations to describe hbs in diverse geometric environments. Vargas et al. ${ }^{14,42}$ have recently evaluated the association energies of these dimers employing MP2 and large basis sets, which provides reliable data for comparing our results.

\section{Computational Aspects}

The association energies of diverse hydrogen-bonded dimers are calculated by subtracting the total energy of the fully relaxed isolated molecules constituting the dimer from the total energy of the fully relaxed hydrogen-bonded dimer. The total energies

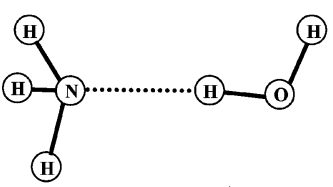

a)

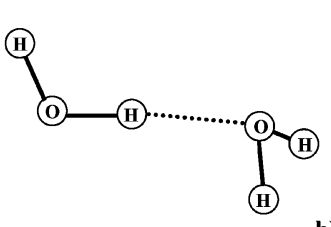

b)

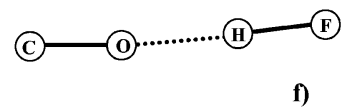

c)
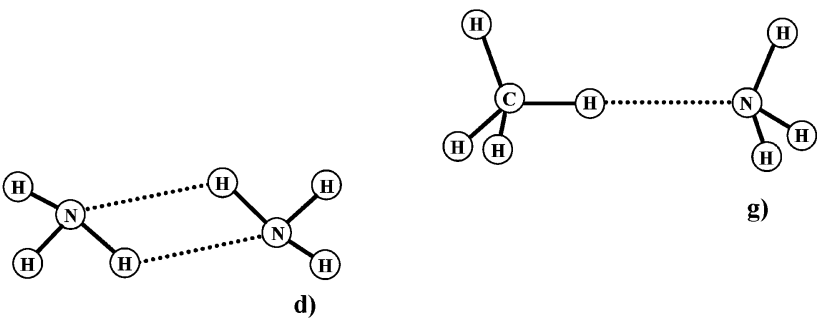

g)

Figure 2. Small hydrogen bonded dimers: (a) $\mathrm{H}_{2} \mathrm{O} \cdots \mathrm{NH}_{3}$ dimer; (b) $\mathrm{H}_{2} \mathrm{O} \cdots \mathrm{H}_{2} \mathrm{O}$ dimer; (c) HF $\cdots \mathrm{HF}$ dimer; (d) $\mathrm{NH}_{3} \cdots \mathrm{NH}_{3}$ dimer; (e) $\mathrm{HCl} \cdots \mathrm{HCl}$ dimer; (f) $\mathrm{CO} \cdots \mathrm{HF}$ dimer; and (g) $\mathrm{CH}_{4} \cdots \mathrm{NH}_{3}$ dimer. Dotted lines denote the hbs.
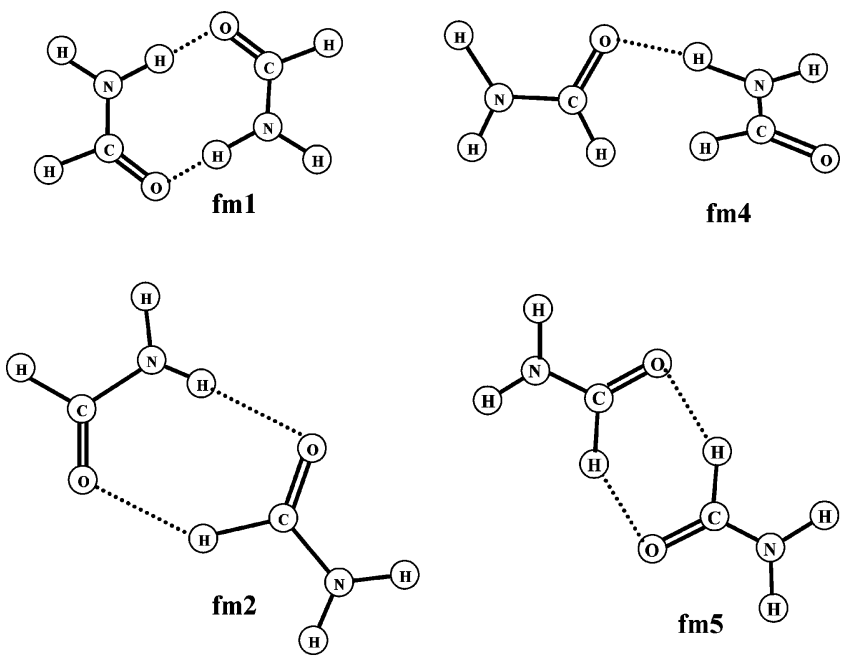

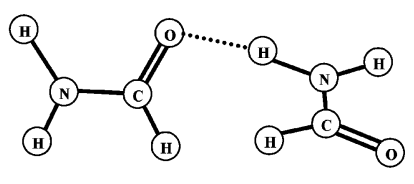

fm4

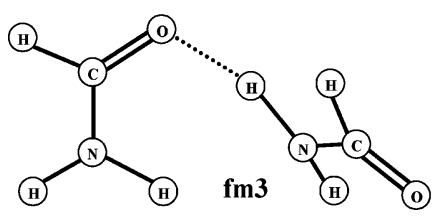

Figure 3. Formamide (fm) dimers. Dotted lines denote the hbs.

are obtained by performing DFT calculations with the PBE functional. We use ab initio pseudopotentials, ${ }^{43}$ plane-wave basis set, and periodic boundary conditions for integrating the KS equations. The calculations have been performed with an energy cutoff of 70 Ry in orthorhombic supercells and with a vacuum 


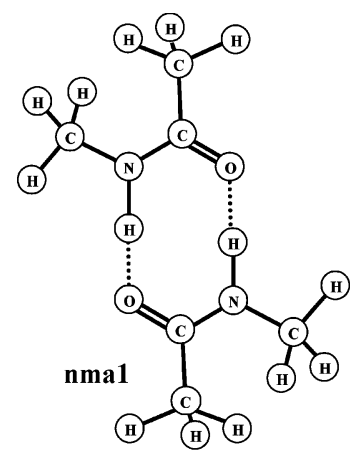

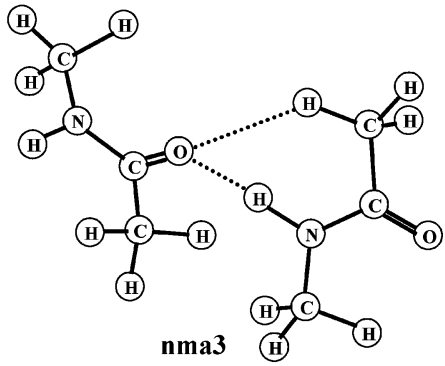

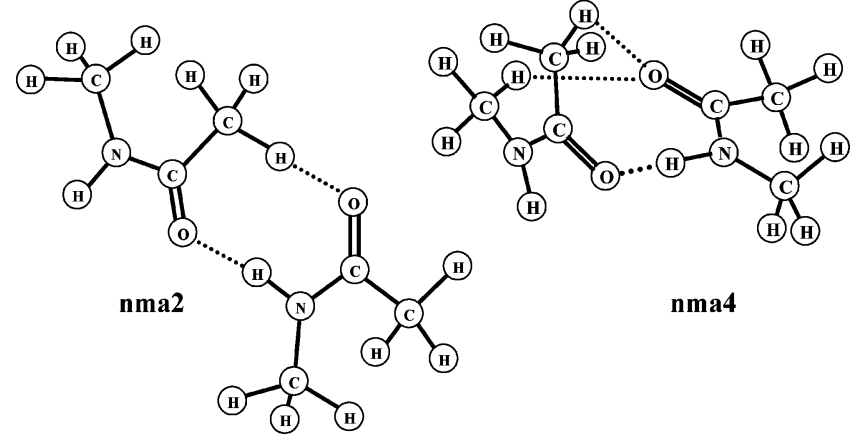

Figure 4. $N$-Methylacetamide (nma) Dimers. Dotted lines denote the hbs.<smiles>CC(C)OC(=O)CCC(=O)OC(C)C(C)C</smiles>

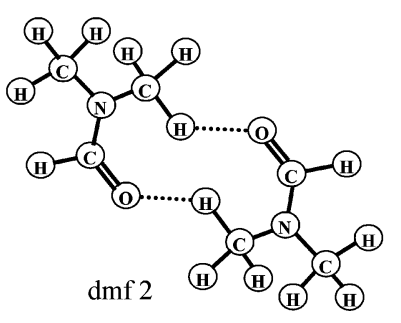

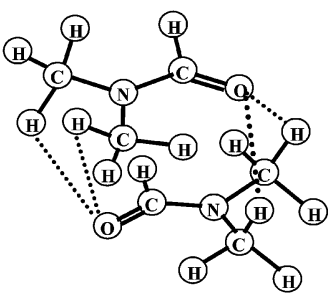

dmf 4

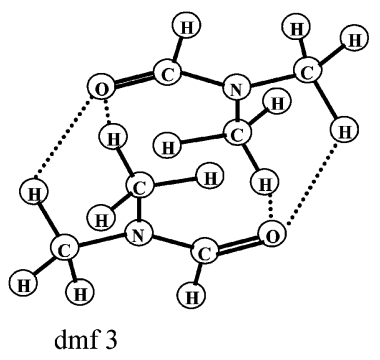

Figure 5. $N, N$-Dimethylformamide (dmf) dimers. Dotted lines denote the hbs.

region of at least $6 \AA$ along the three axes. The Brillouin zone has been sampled at the $\Gamma$-point. All geometry parameters of the studied systems are fully relaxed by searching for the minimum of the total energy. Since many of the systems studied have a large dipole moment (up to $8 \mathrm{D}$ ), total energies are corrected introducing a dipole compensation layer in the middle
TABLE 1: Association Energies (in kcal/mol) for Medium-weak Hydrogen-Bonded Dimers ${ }^{a}$

\begin{tabular}{lclcr}
\hline \multicolumn{1}{c}{ dimer } & hbs & best value & PBE & $\begin{array}{c}\text { PBE error } \\
\text { per hb }\end{array}$ \\
\hline $\mathrm{H}_{2} \mathrm{O} \cdots \mathrm{NH}_{3}$ & 1 & $-6.36^{b}$ & -7.15 & -0.79 \\
$\mathrm{H}_{2} \mathrm{O} \cdots \mathrm{H}_{2} \mathrm{O}$ & 1 & $-4.96^{c}$ & -5.37 & -0.41 \\
$\mathrm{HF} \cdots \mathrm{HF}$ & 1 & $-4.53^{c}$ & -5.08 & -0.55 \\
$\mathrm{NH}_{3} \cdots \mathrm{NH}_{3}$ & 2 & $-2.94^{b}$ & -2.74 & 0.10 \\
$\mathrm{HCl} \cdots \mathrm{HCl}$ & 1 & $-1.95^{c}$ & -2.08 & -0.13 \\
$\mathrm{CO} \cdots \mathrm{HF}$ & 1 & $-1.67^{d}$ & -1.37 & 0.30 \\
$\mathrm{CH}_{4} \cdots \mathrm{NH}_{3}$ & 1 & $-0.66^{e}$ & -0.69 & -0.03
\end{tabular}

${ }^{a}$ The corresponding structures are shown in Figure 2. Best value gives the best (with respect to the basis set) available $\operatorname{CCSD}(\mathrm{T})$ value. ${ }^{b}$ Estimated $\mathrm{CCSD}(\mathrm{T}) / \mathrm{cc}-\mathrm{pVXZ}$ for $\mathrm{X}=\mathrm{D}, \mathrm{T}, \mathrm{Q}$ and 5 interaction energies at the basis set limit. ${ }^{20}{ }^{c}$ Extrapolation of the CCSD(T)/augcc-pVXZ for $\mathrm{X}=\mathrm{D}, \mathrm{T}$, and $\mathrm{Q}$ total energies to the complete basis set limit. ${ }^{45}{ }^{d} \operatorname{CCSD}(\mathrm{T}) /$ aug-cc-pVQZ. ${ }^{13}{ }^{e} \mathrm{CCSD}(\mathrm{T}) / \mathrm{cc}-\mathrm{pVQZ} .{ }^{9}$

of the vacuum region. ${ }^{44}$ Also laterally the molecules interact and the corresponding dipole-dipole interaction is evaluated. Corrections to the association energies due to this effect are in the range of $0.1-0.3 \mathrm{kcal} / \mathrm{mol}$.

\section{Results}

In this section we present the results on the association energies and hb geometry parameters corresponding to the fully optimized structures with DFT-PBE. We structured the section by ordering the systems with respect to size. Thus, first we describe the results of the small hydrogen-bonded systems. Later we focus on the association energies and hb geometry parameters of fm dimers, nma dimers, and dmf dimers, respectively.

Small Hydrogen-Bonded Dimers. In Table 1 we present electronic association energies of a small set of hydrogen-bonded systems (Figure 2). These dimers are bonded by a single linear hb, i.e., $\theta \sim 170-180^{\circ}$, except $\left(\mathrm{NH}_{3}\right)_{2}$, which is bonded by two highly bent hbs, i.e., $\theta \sim 122^{\circ}$. We find that DFT-PBE tends to overestimate the association energy for the stronger hbs with respect to reported CC studies ${ }^{9,13,20,45}$ that included singles, doubles, and triples excitations $(\operatorname{CCSD}(\mathrm{T}))$. The deviation between our DFT-PBE results and the CC results is less than $0.8 \mathrm{kcal} / \mathrm{mol}$. For weak hb dimers with strength $<2 \mathrm{kcal} /$ mol as listed in the second half of Table 1, dispersion interactions are expected to be relevant. This may be the reason for DFT-PBE, which does not account for dispersion interactions, to show a clear tendency to underestimate the association energies in these dimers. In Table 2 some of the characteristic geometric parameters for hbs are reported. DFT-PBE predicts hb bond distances with an error bar $<0.1 \AA$, except for the very weak hydrogen-bonded $\mathrm{CH}_{4} \cdots \mathrm{NH}_{3}$ dimer, where the error bar increases up to $0.13 \AA$. For the hb angle the DFT-PBE results deviate by $<4^{\circ}$ from the CC results. From Table 2 it also becomes obvious that PBE underestimates hb distances for the stronger hbs and overestimates it for the weaker ones. This behavior directly correlates with the trend mentioned above for the PBE hb energies. Experimental association energies for some of the systems studied here are available from literature. Curtiss et al. ${ }^{46}$ reported that the association energy for the $\left(\mathrm{H}_{2} \mathrm{O}\right)_{2}$ dimer is $-5.44 \pm 0.7 \mathrm{kcal} / \mathrm{mol}$. However, Feyereisen et al. ${ }^{36}$ pointed out that the method used for Curtiss et al. to estimate the association energy for the $\left(\mathrm{H}_{2} \mathrm{O}\right)_{2}$ involves the use of theoretical models and does not actually give a dissociation energy from the bottom of the potential energy curve, i.e., free of zero-point vibrational energies and thermal corrections. Pine and Howard ${ }^{47}$ reported association energies for the $(\mathrm{HF})_{2}$ and $(\mathrm{HCl})_{2}$ dimers of $-4.56 \pm 0.29$ and $-2.27 \pm 0.29 \mathrm{kcal} / \mathrm{mol}$, respectively. DFT- 
TABLE 2: Geometry Parameters (hb Length in A, Angles in deg) for Medium-Weak Hydrogen-Bonded Dimers ${ }^{a}$

\begin{tabular}{ccclc}
\hline dimer & parameter & best value & PBE & PBE error \\
\hline $\mathrm{H}_{2} \mathrm{O} \cdots \mathrm{NH}_{3}$ & $r_{\mathrm{hb}}$ & $1.974^{b}$ & 1.91 & -0.064 \\
& $r_{2}$ & $2.942^{b}$ & 2.89 & -0.052 \\
& $\theta$ & $174.71^{b}$ & 172.1 & -2.61 \\
$\mathrm{H}_{2} \mathrm{O} \cdots \mathrm{H}_{2} \mathrm{O}$ & $r_{\mathrm{hb}}$ & $1.94^{c}$ & 1.91 & -0.03 \\
& $r_{2}$ & $2.89^{c}$ & 2.88 & -0.01 \\
& $\theta$ & $172.9^{c}$ & 171.1 & -1.8 \\
& $\sigma$ & $110.5^{c}$ & 107.1 & -3.4 \\
$\mathrm{HF} \cdots \mathrm{HF}$ & $r_{\mathrm{hb}}$ & $1.81^{c}$ & 1.77 & -0.04 \\
& $r_{2}$ & $2.72^{c}$ & 2.71 & -0.01 \\
& $\theta$ & $171.6^{c}$ & 171.0 & -0.6 \\
& $\sigma$ & $115.6^{c}$ & 111.6 & -4.0 \\
$\mathrm{NH}_{3} \cdots \mathrm{NH}_{3}$ & $r_{\mathrm{hb}}$ & $2.472^{b}$ & $2.53^{d}$ & 0.058 \\
& $r_{2}$ & $3.132^{b}$ & $3.18^{d}$ & 0.048 \\
& $\theta$ & & $121.9^{d}$ & \\
& $\alpha$ & $41.92^{b}$ & $42.3^{d}$ & 0.38 \\
$\mathrm{HCl} \cdots \mathrm{HCl}$ & $r_{\mathrm{hb}}$ & $2.53^{c}$ & 2.44 & -0.09 \\
& $r_{2}$ & $3.79^{c}$ & 3.74 & -0.05 \\
& $\theta$ & $169.8^{c}$ & 171.9 & 2.1 \\
& $\sigma$ & $91.7^{c}$ & 91.6 & -0.1 \\
$\mathrm{CO} \cdots \mathrm{HF}$ & $r_{2}$ & $2.9517^{e}$ & 3.01 & 0.0583 \\
& $\theta$ & & & \\
$\mathrm{CH}{ }_{4} \cdots \mathrm{NH} H_{3}$ & $r_{\mathrm{hb}}$ & $2.671^{b}$ & 2.79 & 0.119 \\
& $r_{2}$ & $3.758^{b}$ & 3.89 & 0.132 \\
& $\theta$ & & 179.1 & \\
& $\sigma$ & $112.50^{b}$ & 115.6 & 3.1
\end{tabular}

${ }^{a}$ The structures are shown in Figure 2. The structural parameters are defined in Figure 1. Best value gives the best available value. ${ }^{b}$ Best value, Mp2/cc-pVTZ optimized geometry. ${ }^{9}{ }^{c}$ Best value, estimated in this work by using $\operatorname{CCSD}(\mathrm{T}) /$ aug-cc-pVTZ optimized geometries reported in ref $45 .{ }^{d}$ Our geometry optimization shows the two hbs to be identical. ${ }^{e}$ Best value, CCSD(T)/aug-cc-pVQZ. ${ }^{13}$

PBE results agree with these values within an error of $1 \mathrm{kcal} /$ mol. The magnitude of these DFT-PBE errors seems to be acceptable, and in summary of this part we can conclude that DFT-PBE describes adequately these moderate to weakly hydrogen-bonded dimers. This conclusion applies even for the $\left(\mathrm{NH}_{3}\right)_{2}$ dimer where hbs are highly bent.

To test whether these conclusions are affected by the quality of the basis set we check the convergence of the results obtained with plane-wave basis set with respect to the unit cell size and energy cutoff parameter. We have performed these checks and we verified that the calculated association energies are converged within an error bar of $\sim 0.1 \mathrm{kcal} / \mathrm{mol}$. For example, for $\left(\mathrm{NH}_{3}\right)_{2}$ and $\mathrm{CH}_{4} \cdots \mathrm{NH}_{3}$ dimers the association energies with a $110 \mathrm{Ryd}$ cutoff are -2.89 and $-0.73 \mathrm{kcal} / \mathrm{mol}$, respectively.

Formamide Dimers. Fm dimers are bonded by $\mathrm{N}-\mathrm{H} \cdots \mathrm{O}$ and/or $\mathrm{CH} \cdots \mathrm{O}$ hbs (Figure 3). These hbs are relevant because they are structurally similar to the most ubiquitous hbs found in proteins. Therefore they are suitable models to estimate the DFT accuracy to describe hbs in proteins. Recently, Vargas et al. ${ }^{42}$ evaluated the association energies of these fm dimers by extrapolating MP2 results to the basis set limit. This was achieved by using augmented correlation-consistent high-level basis sets (aug-cc-pVXZ, where $\mathrm{X}=\mathrm{D}, \mathrm{T}$, or $\mathrm{Q}$ ). Results obtained by extrapolating MP2 results to the basis set limit agree well with $\operatorname{CCSD}(\mathrm{T})$ results as has been shown, e.g., for the $\left(\mathrm{H}_{2} \mathrm{O}\right)_{2}$ dimer. $^{36}$ As is obvious from Table 3 DFT-PBE association energies are in excellent agreement with the results reported by Vargas et al.: the error bar is $<1 \mathrm{kcal} / \mathrm{mol}$. The only exception is found for the weakly bonded fm5 dimer where the error bar is $\sim 1.3 \mathrm{kcal} / \mathrm{mol}$. However, for the hb strength (i.e., the association energy divided per number of hbs) the DFTPBE error is only $\sim 0.7 \mathrm{kcal} / \mathrm{mol}$ (Table 3 ). We note that the sign of the error in the association energies is opposite for these dimers and the set of small dimers discussed earlier: DFT-PBE
TABLE 3: Association Energies (in $\mathrm{kcal} / \mathrm{mol}$ ) for Formamide (fm) Hydrogen-Bonded Dimers ${ }^{a}$

\begin{tabular}{ccccc}
\hline dimer & hbs & best value & PBE & $\begin{array}{c}\text { PBE error } \\
\text { per hb }\end{array}$ \\
\hline $\mathrm{fm} 1$ & 2 & -14.35 & $-14.34^{b}$ & 0.01 \\
$\mathrm{fm} 2$ & 2 & -9.70 & $-9.11^{b}$ & 0.30 \\
$\mathrm{fm} 3$ & 1 & -7.34 & $-6.78^{b}$ & 0.56 \\
$\mathrm{fm} 4$ & 1 & -6.76 & $-6.28^{b}$ & 0.48 \\
$\mathrm{fm} 5$ & 2 & -5.02 & -3.68 & 0.67
\end{tabular}

${ }^{a}$ The corresponding structures are shown in Figure 3. Best value corresponds to extrapolation of the MP2/aug-cc-pVXZ for $\mathrm{X}=\mathrm{D}, \mathrm{T}$, and Q total energies to the complete basis set limit. ${ }^{42}{ }^{b} \mathrm{PBE}$ values from ref 30 .

TABLE 4: Geometry Parameters (hb Length in Å, Angles in deg) for Formamide (fm) Hydrogen-Bonded Dimers ${ }^{a}$

\begin{tabular}{|c|c|c|c|c|c|c|c|}
\hline \multirow[b]{2}{*}{ dimer } & \multirow[b]{2}{*}{ parameter } & \multicolumn{2}{|c|}{ best value } & \multicolumn{2}{|c|}{ PBE } & \multicolumn{2}{|c|}{ PBE error } \\
\hline & & $\mathrm{NH} \cdots \mathrm{O}$ & $\mathrm{CH} \cdots \mathrm{O}$ & $\mathrm{NH} \cdots \mathrm{O}$ & $\mathrm{CH} \cdots \mathrm{O}$ & $\mathrm{NH} \cdots \mathrm{O}$ & $\mathrm{CH} \cdots \mathrm{C}$ \\
\hline \multirow[t]{4}{*}{$\mathrm{fm} 1$} & $r_{\mathrm{hb}}$ & 1.825 & & $1.81^{b}$ & & -0.015 & \\
\hline & $r_{2}$ & 2.842 & & 2.85 & & 0.008 & \\
\hline & $\theta$ & 174.2 & & $174.0^{b}$ & & -0.2 & \\
\hline & $\sigma$ & 120.1 & & $120.2^{b}$ & & 0.1 & \\
\hline \multirow[t]{4}{*}{$\mathrm{fm} 2$} & $r_{\mathrm{hb}}$ & 1.857 & 2.234 & $1.84^{c}$ & $2.28^{c}$ & -0.017 & 0.046 \\
\hline & $r_{2}$ & 2.842 & 3.190 & 2.86 & 3.23 & 0.018 & 0.04 \\
\hline & $\theta$ & 168.9 & 144.9 & $168.9^{c}$ & $142.6^{c}$ & 0.0 & -2.3 \\
\hline & $\sigma$ & 105.7 & 113.1 & $107.3^{c}$ & $113.8^{c}$ & 1.6 & 0.7 \\
\hline \multirow[t]{4}{*}{$\mathrm{fm} 3$} & $r_{\mathrm{hb}}$ & 1.935 & & $1.92^{b}$ & & -0.015 & \\
\hline & $r_{2}$ & 2.902 & & 2.91 & & 0.008 & \\
\hline & $\theta$ & 158.4 & & $160.8^{b}$ & & 2.4 & \\
\hline & $\sigma$ & 110.3 & & $113.0^{b}$ & & 2.7 & \\
\hline \multirow[t]{4}{*}{$\mathrm{fm} 4$} & $r_{\mathrm{hb}}$ & 1.904 & & $1.90^{b}$ & & -0.004 & \\
\hline & $r_{2}$ & 2.894 & & 2.91 & & 0.016 & \\
\hline & $\theta$ & 165.8 & & $166.3^{b}$ & & 0.5 & \\
\hline & $\sigma$ & 108.9 & & $110.9^{b}$ & & 2.0 & \\
\hline \multirow[t]{4}{*}{ fm5 } & $r_{\mathrm{hb}}$ & & 2.320 & & 2.36 & & 0.04 \\
\hline & $r_{2}$ & & 3.271 & & 3.31 & & 0.039 \\
\hline & $\theta$ & & 144.4 & & 142.7 & & -1.7 \\
\hline & $\sigma$ & & 93.3 & & 94.7 & & 1.4 \\
\hline
\end{tabular}

${ }^{a}$ The structures are shown in Figure 3. The structural parameters are defined in Figure 1. Best value corresponds to MP2/aug-cc-pVTZ optimized geometry. ${ }^{42}$ Our geometry optimization shows the two hbs to be identical in the fm1 and fm5 dimers. ${ }^{b} \mathrm{PBE}$ values were taken from ref $30 .{ }^{c}$ PBE values were taken from ref 48 .

underestimates the hb strength for fm dimers and overestimates it for the small ones. A possible explanation is that the benchmark calculations used to compare against DFT rely on two different approaches: the small dimers are compared against CC results while the fm dimers are compared against MP2 results. Regarding the hb geometry parameters for the fm dimers, our DFT-PBE results are in excellent agreement with those calculated by MP2 (Table 4). Specifically, the error bar for hb distances is $<0.05 \AA$. Likewise, hb angles are described by DFT$\mathrm{PBE}$ with an error bar $<3^{\circ}$. Therefore, we can conclude that DFT-PBE describes adequately medium to weak hbs, as are characteristic for fm dimers. Comparison of our results against experiments is not possible for the fm dimers because the experimental association energies in the gas phase for such systems have not been determined, and in the fm crystal $^{49}$ structure each fm monomer forms four hbs, rather than one or two as in the dimers studied here.

$\mathrm{N}$-Methylacetamide Dimers. Another set of dimers bonded by $\mathrm{N}-\mathrm{H} \cdots \mathrm{O}$ and/or by $\mathrm{CH} \cdots \mathrm{O}$ hbs are the nma complexes (Figure 4). These dimers have been studied by Vargas et al. ${ }^{42}$ employing the MP2 method (MP2/aug-cc-pVTZ single-point energy calculations on MP2/aug-cc-pVDZ optimized geometry). A comparison with our results shows DFT-PBE to strongly underestimate association energies (Table 5). The error bar is $\sim 2 \mathrm{kcal} / \mathrm{mol}$ for $\mathrm{nma} 1$ and nma2 dimers but increases for nma3 
TABLE 5: Association Energies (in $\mathrm{kcal} / \mathrm{mol}$ ) for $N$-Methylacetamide (nma) Hydrogen-Bonded Dimers ${ }^{a}$

\begin{tabular}{ccccc}
\hline dimer & hbs & best value & PBE & $\begin{array}{c}\text { PBE error } \\
\text { per hb }\end{array}$ \\
\hline nma1 & 2 & -17.18 & $-15.27^{b}$ & 0.96 \\
nma2 & 2 & -10.76 & $-8.57^{b}$ & 1.10 \\
nma3 & 2 & -9.67 & -7.1 & 1.29 \\
nma4 & 3 & -12.37 & -8.1 & 1.42
\end{tabular}

${ }^{a}$ The corresponding structures are shown in Figure 4. Best value corresponds to MP2/aug-cc-pVTZ single point energy calculation on the MP2/aug-cc-pVDZ optimized geometry. ${ }^{42} \quad{ }^{b} \mathrm{PBE}$ values from ref 30 .

and nma4 dimers. Actually, for the nma4 dimer the largest error in the association energy in this study is found ( $\sim 4 \mathrm{kcal} / \mathrm{mol})$. However, since the number of hbs in this dimer is 3 , the DFT$\mathrm{PBE}$ error bar per hb reduces to $\sim 1.4 \mathrm{kcal} / \mathrm{mol}$. As shown in Table 6 DFT-PBE error bars for hb geometry parameters increase with respect to those for fm dimers: the error in hb distances is $<0.3 \AA$, errors in hb angles are $<3^{\circ}$. An exception is found for the nma3 dimer where the differences between DFTPBE and MP2 increase up to $24^{\circ}$. We further note that the biggest DFT-PBE errors for hb geometry parameters correspond to $\mathrm{CH} \cdots \mathrm{O}$ hbs in nma3 and nma4 complexes. Interestingly, both dimers also have highly bent hbs; i.e., $\theta \sim 120^{\circ}$. For the nma dimers association energies in the gas phase have not been determined. Recently Kearley et al. ${ }^{50}$ have determined the crystal structure of trans-nma at $2 \mathrm{~K}$ using powder neutron diffraction on methyl-deuterated material. They report for the $\mathrm{NH} \cdots \mathrm{O} h b$, $r_{\mathrm{hb}}=1.946 \AA$ and $\theta=179.0^{\circ}$. The nma3 dimer is formed by trans-isomers of nma. Interestingly the DFT-PBE geometry for the $\mathrm{NH} \cdots \mathrm{O}$ hb in nma3 is closer to the experimental one than the corresponding MP2 geometry. However, care should be taken in such comparison: in the crystal the nma molecule seems to be part of a one-dimensional hydrogen-bonded chain, where a cooperative effect may be present, and in the nma3 dimer this is absent.

$\mathrm{N}, \boldsymbol{N}$-Dimethylformamide Dimers. Dmf dimers are bonded only by $\mathrm{CH} \cdots \mathrm{O}$ hbs (Figure 5 ). Therefore, these dimers are good models to determine whether the large DFT-PBE errors found for the nma dimers are associated to a wrong description of $\mathrm{CH} \cdots \mathrm{O}$ hbs by DFT-PBE or to other factors such as the presence of bent hbs. Vargas et al. ${ }^{14}$ studied these dimers using MP2/ aug-cc-pVTZ single-point calculations on geometries obtained with a DZP basis set plus diffuse functions (DZP+diff). Comparing against results by Vargas et al. we find that DFTPBE (Table 7) underestimates the association energies for $\mathrm{dmf} 1$ and dmf 2 by $\sim 1.5 \mathrm{kcal} / \mathrm{mol}$. However, DFT-PBE strongly underestimates the association energies for $\mathrm{dmf} 3$ and $\mathrm{dmf} 4$ dimers by $\sim 5 \mathrm{kcal} / \mathrm{mol}$. The DFT-PBE error per hb is less drastic; it underestimates each hb present in the dimer by $\sim 1$ $\mathrm{kcal} / \mathrm{mol}$. In Table 8 we reported the hb geometrical parameters for the dmf dimers. DFT-PBE deviates from the MP2 results by about $\sim 0.35 \AA$ in distances and by $\sim 5^{\circ}$ in angles. The stronger deviations correspond to $\mathrm{dmf} 3$ and $\mathrm{dmf} 4$ dimers where the largest energy discrepancies are observed as well. Also, these dimers form highly bent hbs, i.e., the $\theta$ angle is in the range between $120^{\circ}$ and $130^{\circ}$. As for the fm and nma dimers, for the $\mathrm{dmf}$ dimers the association energies in the gas phase have not been determined. Moreover, in the dmf crystal ${ }^{51}$ a four-member ring of dmf molecules connected by hbs is formed, a situation that any of the dimers studied here fulfill.

\section{Discussion}

Several factors should be taken into account to determine the performance of a functional to describe hbs, i.e., the kind of hb studied, the basis set used, and the accuracy of the values used as reference for the comparison. Although DFT-PBE describes adequately the different types of hbs studied here, a feature characteristic to all systems exhibiting large errors in association energies and geometries is the presence of $\mathrm{CH} \cdots \mathrm{O}$ hbs. However, a detailed analysis of the hb geometries shows that the DFT-PBE errors are not intrinsic to the presence of $\mathrm{CH} \cdots \mathrm{O}$ hbs but they occur whenever the structure exhibits a sizable deviation of the $\theta$ angle from linearity. In Figure 6 the DFT-PBE error for the hb strength with respect to the $\theta$ angle is shown. For dimers with several hbs we choose the smallest value for $\theta$, i.e., the $\theta$ value that exhibits the largest deviation from the ideal linear $\left(180^{\circ}\right)$ angle. This plot reveals a clear trend: the higher the hb deviation from linearity (i.e., the smaller $\theta$ ), the larger are the DFT errors for the hb strength. This finding explains why DFT-PBE fails to describe the $\mathrm{dmf} 3$ and $\mathrm{dmf} 4$, nma3 and nma4 $\mathrm{CH} \cdots \mathrm{O}$ bonded dimers, where the $\theta$ angle is $<130^{\circ}$. It also explains why other dimers containing $\mathrm{CH} \cdots \mathrm{O}$ hbs, where $\theta$ is $>130^{\circ}$, are well-described by DFT-PBE, e.g. $\mathrm{fm} 2, \mathrm{fm} 5, \mathrm{nma} 2, \mathrm{dmf} 1$, and $\mathrm{dmf} 2$. Our finding also includes the DFT-PBE errors for $\mathrm{NH} \cdots \mathrm{O}$ hbs. For example, for the nma3

TABLE 6: Geometry Parameters (hb Length in $\AA$, Angles in deg) for $N$-Methylacetamide (nma) Hydrogen-Bonded Dimers ${ }^{a}$

\begin{tabular}{|c|c|c|c|c|c|c|c|c|c|c|}
\hline \multirow[b]{3}{*}{ dimer } & \multirow[b]{3}{*}{ parameter } & \multicolumn{3}{|c|}{ best value } & \multicolumn{3}{|c|}{ PBE } & \multicolumn{3}{|c|}{ PBE error } \\
\hline & & \multirow[b]{2}{*}{$\mathrm{N}-\mathrm{H} \cdots \mathrm{O}$} & \multicolumn{2}{|c|}{$\mathrm{C}-\mathrm{H} \cdots \mathrm{O}$} & \multirow[b]{2}{*}{$\mathrm{N}-\mathrm{H} \cdots \mathrm{O}$} & \multicolumn{2}{|c|}{$\mathrm{C}-\mathrm{H} \cdots \mathrm{O}$} & \multirow[b]{2}{*}{$\mathrm{N}-\mathrm{H} \cdots \mathrm{O}$} & \multicolumn{2}{|c|}{$\mathrm{C}-\mathrm{H} \cdots \mathrm{O}$} \\
\hline & & & s-hb & 1-hb & & s-hb & 1-hb & & s-hb & 1-hb \\
\hline \multirow{4}{*}{ nma1 } & $r_{\mathrm{hb}}$ & 1.799 & & & $1.78^{b}$ & & & -0.019 & & \\
\hline & $r_{2}$ & 2.832 & & & 2.82 & & & -0.012 & & \\
\hline & $\theta$ & 177.7 & & & $178.1^{b}$ & & & 0.4 & & \\
\hline & $\sigma$ & 118.6 & & & $119.5^{b}$ & & & 0.9 & & \\
\hline \multirow[t]{4}{*}{ nma2 } & $r_{\mathrm{hb}}$ & 1.867 & 2.249 & & $1.87^{b}$ & $2.25^{b}$ & & 0.003 & 0.001 & \\
\hline & $r_{2}$ & 2.881 & 3.346 & & 2.89 & 3.35 & & 0.009 & 0.004 & \\
\hline & $\theta$ & 169.0 & 177.4 & & $169.9^{b}$ & $176.0^{b}$ & & 0.9 & -1.4 & \\
\hline & $\sigma$ & 122.2 & 117.4 & & $123.2^{b}$ & $118.6^{b}$ & & 1.0 & 1.2 & \\
\hline \multirow[t]{4}{*}{ nma3 } & $r_{\mathrm{hb}}$ & 2.035 & 2.685 & & 1.95 & 2.64 & & -0.085 & -0.045 & \\
\hline & $r_{2}$ & 2.949 & 3.347 & & 2.97 & 3.55 & & 0.021 & 0.203 & \\
\hline & $\theta$ & 148.1 & 118.3 & & 170.4 & 139.9 & & 22.3 & 21.6 & \\
\hline & $\sigma$ & 106.3 & 91.4 & & 122.4 & 115.4 & & 16.1 & 24.0 & \\
\hline \multirow{4}{*}{ nma4 } & $r_{\mathrm{hb}}$ & 1.904 & 2.496 & 2.527 & 1.93 & 2.73 & 2.68 & 0.026 & 0.234 & 0.153 \\
\hline & $r_{2}$ & 2.919 & 3.273 & 3.211 & 2.95 & 3.46 & 3.37 & 0.031 & 0.187 & 0.159 \\
\hline & $\theta$ & 171.1 & 126.5 & 119.2 & 172.3 & 124.0 & 120.7 & 1.2 & -2.5 & 1.5 \\
\hline & $\sigma$ & 101.0 & 163.6 & 120.9 & 103.5 & 161.1 & 120.0 & 2.5 & -2.5 & -0.9 \\
\hline
\end{tabular}

${ }^{a}$ The structures are shown in Figure 4. The structural parameters are defined in Figure 1. s-hb and l-hb stand for short-hb and long-hb, respectively. Best value corresponds to MP2/aug-cc-pVDZ optimized geometry. ${ }^{42}{ }^{b}$ PBE values were taken from ref 30. 
TABLE 7: Association Energies (in $\mathrm{kcal} / \mathrm{mol}$ ) for $N, N$-Dimethylformamide (dmf) Hydrogen-Bonded Dimers ${ }^{a}$

\begin{tabular}{ccccc}
\hline dimer & hbs & best value & PBE & $\begin{array}{c}\text { PBE error } \\
\text { per hb }\end{array}$ \\
\hline dmf1 & 2 & -5.35 & -3.80 & 0.78 \\
dmf2 & 2 & -4.14 & -2.81 & 0.67 \\
dmf3 & 4 & -8.34 & -3.76 & 1.15 \\
dmf4 & 4 & -8.90 & -4.09 & 1.20
\end{tabular}

${ }^{a}$ The corresponding structures are shown in Figure 5. Best value corresponds to MP2/aug-cc-pVTZ single-point calculation on MP2/ DZP+dif geometries. ${ }^{14}$

TABLE 8: Geometry Parameters (hb Length in Å, Angles in $\mathrm{deg}$ ) for $N, N$-Dimethylformamide (dmf) Hydrogen-Bonded Dimers $^{a}$

\begin{tabular}{|c|c|c|c|c|c|c|c|}
\hline \multirow[b]{2}{*}{ dimer } & \multirow[b]{2}{*}{ parameter } & \multicolumn{2}{|c|}{ best value } & \multicolumn{2}{|c|}{ PBE } & \multicolumn{2}{|c|}{ PBE error } \\
\hline & & s-hb & $1-\mathrm{hb}$ & s-hb & 1-hb & s-hb & $1-h b$ \\
\hline \multirow[t]{4}{*}{$\mathrm{dmf1}$} & $r_{\mathrm{hb}}$ & 2.36 & & 2.43 & & 0.07 & \\
\hline & $r_{2}$ & 3.32 & & 3.40 & & 0.08 & \\
\hline & $\theta$ & 146.0 & & 143.8 & & -2.2 & \\
\hline & $\sigma$ & 92.0 & & 93.7 & & 1.7 & \\
\hline \multirow[t]{4}{*}{$\mathrm{dmf} 2$} & $r_{\mathrm{hb}}$ & 2.36 & & 2.47 & & 0.11 & \\
\hline & $r_{2}$ & 3.29 & & 3.40 & & 0.11 & \\
\hline & $\theta$ & 143.0 & & 141.8 & & -1.2 & \\
\hline & $\sigma$ & 145.0 & & 144.0 & & -1.0 & \\
\hline \multirow[t]{4}{*}{$\mathrm{dmf} 3$} & $r_{\mathrm{hb}}$ & 2.52 & 2.87 & 2.79 & 3.14 & 0.27 & 0.27 \\
\hline & $r_{2}$ & 3.25 & 3.48 & 3.54 & 3.79 & 0.29 & 0.31 \\
\hline & $\theta$ & 123.0 & 115.0 & 125.3 & 118.6 & 2.3 & 3.6 \\
\hline & $\sigma$ & 125.0 & 79.0 & 119.8 & 77.1 & -5.2 & -1.9 \\
\hline \multirow[t]{4}{*}{$\mathrm{dmf} 4$} & $r_{\mathrm{hb}}$ & 2.55 & 2.59 & 2.67 & 2.92 & 0.12 & 0.33 \\
\hline & $r_{2}$ & 3.32 & 3.32 & 3.49 & 3.66 & 0.17 & 0.34 \\
\hline & $\theta$ & 127.0 & 124.0 & 130.5 & 124.2 & 3.5 & 0.2 \\
\hline & $\sigma$ & 111.0 & 103.0 & 114.2 & 101.0 & 3.2 & -20 \\
\hline
\end{tabular}

${ }^{a}$ The structures are shown in Figure 5. The structural parameters are defined in Figure 1. s-hb and l-hb stand for short-hb and long-hb, respectively. Best value corresponds to MP2/DZP+diff optimized geometry. ${ }^{14}$ Our geometry optimization shows the two hbs to be identical in $\mathrm{dmf} 1$ and dmf2. Our geometry optimization shows for $\mathrm{dmf} 3$ and dmf 4 the two s-hbs to be identical; it also applies to the two l-hbs.

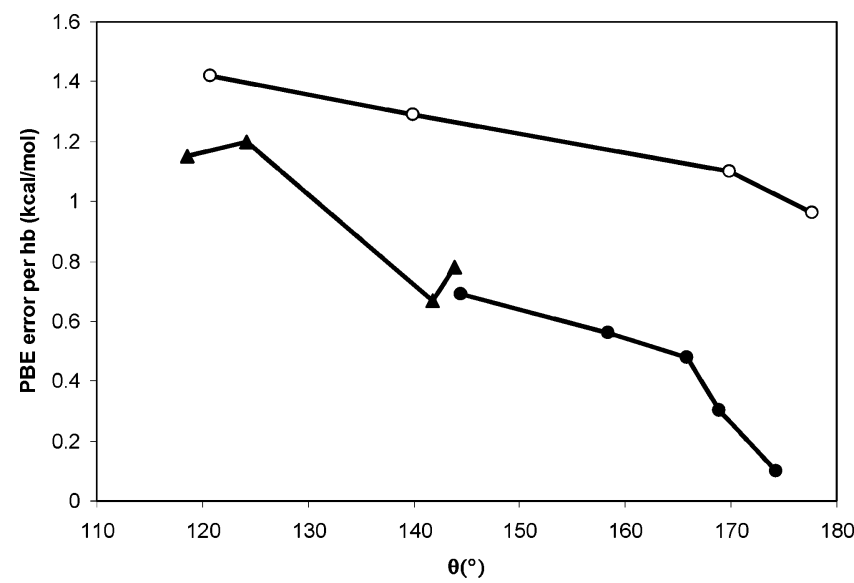

Figure 6. DFT-PBE error for the description of the hb strength as a function of the hb directionality. Close circles stand for fm dimers. Open circles stand for nma dimers. Close triangles stand for dmf dimers. Solid lines are drawn as a guide to the eye.

dimer where the $\theta$ angle of the $\mathrm{CH} \cdots \mathrm{O}$ hb is $<130^{\circ}$, we find the largest errors on the DFT-PBE geometry for the $\mathrm{NH} \cdots \mathrm{O}$ hb with respect to MP2 results. For this dimer MP2 predicts a value for the $\theta$ angle of the $\mathrm{NH} \cdots \mathrm{O}$ hb of $\sim 150^{\circ}$, which DFTPBE predicts to be almost linear, i.e., $\theta \sim 170^{\circ}$. This is a somewhat unexpected result, as typically DFT-PBE gives quite good results for geometries. We note that the potential energy surface for that dimer is quite flat: the DFT-PBE energy for the nma3 dimer in the MP2 geometry is only $4 \mathrm{kcal} / \mathrm{mol}$ higher than the DFT-PBE energy for the fully relaxed DFT-PBE geometry, i.e., $\sim 0.2 \mathrm{kcal} / \mathrm{mol}$ per degree of deviation of the $\theta$ angle. This might be explained by the fact that DFT-PBE predicts a very weak interaction for the highly bent $\mathrm{CH} \cdot \cdots \mathrm{O}$ $\mathrm{hb}$. Then the stronger $\mathrm{N}-\mathrm{H} \cdots \mathrm{O}$ hb dominates and distorts the dimer structure (with respect to the MP2 one) toward a more linear $\mathrm{N}-\mathrm{H} \cdots \mathrm{O}$ hb. The DFT-PBE geometry for this hb is in good agreement with that determined experimentally in the nma3 crystal. However, this may be a fortuitous result. Notwithstanding, the large discrepancies between DFT-PBE and MP2 geometries in the nma3 dimer correlate with the presence of a highly bent $\mathrm{hb}$.

The trend between the DFT-PBE error and the hb directionality identified here may be related to the insufficient description of the dispersion interaction (i.e., the attraction due to fluctuations in the charge density). This can be seen by noting that for highly nonlinear hbs, electrostatic interactions such as chargedipole and dipole-dipole are much less prominent than in a linear conformation. This effect reduces the total hb strength and makes dispersion interaction a more relevant contribution. In fact, the above scenario clearly relates to a problem pointed out by a number of authors, namely how to define when a hb transforms to another kind of interaction such as, e.g., into a vdW interaction. ${ }^{1,4,5}$ Novoa et al. ${ }^{4}$ have shown that for $\mathrm{CH} \cdots \mathrm{O}$ hbs vdW interactions become dominant with increasing deviation of the hb from linearity. Therefore, the failure of DFT$\mathrm{PBE}$ to describe highly bent hbs is mainly caused by the inability of present day DFT functionals to correctly describe the dispersion energy.

Finally, it should be mentioned that discrepancies between DFT and MP2 cannot be solely attributed to DFT errors. Figure 6 clearly indicates that the DFT-PBE error bar decreases when the quality of the basis set used in MP2 calculations increases. It is particularly noteworthy that when the comparison is restricted to fm dimers, where the extrapolation to the basis set limit has been applied, the DFT-PBE error per hb is systematically smaller than $1 \mathrm{kcal} / \mathrm{mol}$. Therefore, at the moment we cannot rule out that the larger deviations observed for the nma and dmf dimers might have as well contributions from errors due to the incompleteness of the basis set used in the MP2 calculations.

\section{Conclusions}

On the basis of the above results we conclude DFT-PBE to predict the strength of hbs with an accuracy of $1 \mathrm{kcal} / \mathrm{mol}$. Exceptions occur for cases where hbs are highly bent, i.e., for $\theta$ angles $<130^{\circ}$ : for these structures the error can increase to as much as $1.5 \mathrm{kcal} / \mathrm{mol}$ per hb. The DFT-PBE description of geometry parameters for hbs follows the same trend; they are well-described except where highly bent cases appear. We believe that the clear relation between directionality and $\mathrm{xc}$ functional error bar may serve as a guide to know when DFT calculations may fail to describe hydrogen-bonded systems. Finally, it appears that the discrepancy between DFT and MP2 in the description of hbs arises from the known deficiency of DFT to describe the dispersion energy.

Acknowledgment. J.I. thanks Jorge Garza, Rubicelia Vargas, and Martin Fuchs for helpful discussions.

\section{References and Notes}

(1) Steiner, T. Angew. Chem., Int. Ed. 2002, 41, 48.

(2) Buckingham, A. D. The hydrogen bond: An Electrostatic interaction? Theoretical Treatments of hydrogen bonding; Hadži, D., Ed.; John Wiley \& Sons Ltd: Chichister, UK, 1997. 

511.

3) Perrin, C. L.; Nielson, J. B. Annu. Rev. Phys. Chem. 1997, 48,

(4) Novoa, J. J.; Fuente, P.; Mota, F. Chem. Phys. Lett. 1998, 290, 159.

(5) Steiner, T.; Desiraju, G. R. Chem. Commun. 1998, 891

(6) Müller-Dethlefs, K.; Hobza, P. Chem. Rev. 2000, 100, 143.

(7) Del Bene, J. E. Hydrogen bonding: 1. Encyclopedia of Computational Chemistry; Schleyer, D., Editor in Chief; John Wiley: Chichester, UK, 1998; Vo1. 2.

(8) Del Bene, J. E.; Jordan, M. J. T. J. Mol. Struct. (THEOCHEM)

2001, 573, 11

(9) Rappé, A. K.; Bernstein, E. R. J. Phys. Chem. A 2000, 104, 6117. (10) Sim, F.; St-Amant, A.; Papai, I.; Salahub, D. R. J. Am. Chem. Soc. 1992, 114, 4391.

(11) Laasonen, K.; Csajka, F.; Parrinello, M. Chem. Phys. Lett. 1992 194, 172.

(12) Guo, H.; Sirois, S.; Proynov, E. I.; Salahub, D. R. Density Functional Theory and its Applications to Hydrogen-bonded Systems. Theoretical Treatments of hydrogen bonding; Hadži, D., Ed.; John Wiley \& Sons Ltd: Chichister, UK, 1997.

(13) Tuma, A. C.; Boese, D.; Handy, N. Phys. Chem. Chem. Phys. 1999 $1,3939$.

(14) Vargas, R.; Garza, J.; Dixon, D. A.; Hay, B. P. J. Am. Chem. Soc. 2000, $122,4750$.

(15) Hamman, D. R. Phys. Rev. B 1997, 55, 10157.

(16) Kaschner, R.; Hohl, D. J. Phys. Chem. A 1998, 102, 5111.

(17) Xantheas, S. S. J. Chem. Phys. 1995, 102, 4505.

(18) Lee, C.; Yang, W.; Parr, R. G. Phys. Rev. B 1988, 37, 785.

(19) Becke, A. D. J. Chem. Phys. 1993, 98, 5648.

(20) Tsuzuki, S.; Lüthi, H. P. J. Chem. Phys. 2001, 114, 3949.

(21) Kim, K.; Jordan K. D. J. Phys. Chem. 1994, 98, 10089.

(22) Perdew, J. P.; Wang, Y. Phys. Rev. B 1992, 45, 13244.

(23) Becke, A. Phys. Rev. A 1988, 38, 3098.

(24) Perdew, J. Phys. Rev. B 1986, 33, 8822.

(25) Perdew, J.; Burke, K.; Ernzerhof, M. Phys. Rev. Lett. 1996, 77, 3865 .

(26) Laasonen, K.; Sprik, M.; Parrinello, M. J. Chem. Phys. 1993, 99 , 9080 .

(27) Ernzerhof, M.; Scuseria G. E. J. Chem. Phys. 1999, 110, 5029.

(28) Improta, R.; Barone, V.; Kudin, K. N.; Scuseria, G. E. J. Am. Chem. Soc. 2001, 123, 3311 .
(29) Rovira, C.; Novoa, J.; Ballone, P. J. Chem. Phys. 2001, 115, 6406 (30) Ireta, J.; Neugebauer, J.; Scheffler, M.; Rojo, A.; Galván, M J. Phys. Chem. B 2003, 107, 1432

(31) Proynov, E.; Chermette, H.; Salahub, D. R. J. Chem. Phys. 2000, $113,10013$.

(32) Rabuck, A. D.; Scuseria, G. E. Theor. Chim. Acc. 2000, 104, 439.

(33) Barone, V.; Adamo, C. J. Chem. Phys. 1996, 105, 11007.

(34) Van Duijneveldt-Van de Rijdt, J. G. C. M.; Van Duijneveldt, F. B. Ab initio methods applied to hydrogen-bonded systems. Theoretical Treatments of hydrogen bonding; Hadži, D., Ed.; John Wiley \& Sons Ltd: Chichister, UK, 1997.

(35) Boys, S. F.; Bernardi, F. Mol. Phys. 1970, 19, 553.

(36) Feyereisen, M. W.; Feller, D.; Dixon, D. A. J. Phys. Chem. 1996, 100, 2993.

(37) Delley, B. J. Chem. Phys. 1990, 92, 508

(38) Arstila, H.; Laasonen, K.; Laaksonen, A. J. Chem. Phys. 1998, 108 1031

(39) Payne, M. C.; Teter, M. P.; Allan, D. C.; Arias, T. A.; Joannopoulos, J. D. Rev. Mod. Phys. 1992, 64, 1045.

(40) Bockstedte, M.; Kley, A.; Neugebauer, J.; Scheffler, M. Comput Phys. Commun. 1997, 107, 187.

(41) Fellers, R. S.; Barsky, D.; Gygi, F.; Colvin, M. Chem. Phys. Lett. 1999, 312, 548

(42) Vargas, R.; Garza, J.; Friesner, R. A.; Stern, H.; Hay, B. P.; Dixon,

D. A. J. Phys. Chem. A 2001, 105, 4963.

(43) Troullier, N.; Martins, J. L. Phys. Rev. B 1991, 43, 1993. Fuchs, M.; Scheffler, M. Comput. Phys. Commun. 1999, 119, 67.

(44) Neugebauer, J.; Scheffler, M. Phys. Rev. B 1992, 46, 16067.

(45) Halkier, A.; Klopper, W.; Helgaker, T.; Jørgensen, P.; Taylor, P. R. J. Chem. Phys. 1999, 111, 9157.

(46) Curtiss, L. A.; Frurip, D. J.; Blander, M. J. Chem. Phys. 1979, 71, 2703.

(47) Pine, A. S.; Howard, B. J. J. Chem. Phys. 1986, 84, 590.

(48) Ireta, J.; Neugebauer, J.; Scheffler, M.; Rojo, A.; Galván, M J Phys. Chem. B 2003, 107, 9616.

(49) Ladell, J.; Post, B. Acta Crystallogr. B 1954, 7, 559.

(50) Kearley, G. J.; Johnson, M. R.; Plazanet, M.; Suard, E. J. Chem. Phys. 2001, 115, 2614

(51) Borrmann, H.; Persson, I.; Sandström, M.; Stålhandske, C. M. V. J. Chem. Soc., Perkin Trans. 2000, 2, 393 